\title{
Lbx1 Acts as a Selector Gene in the Fate Determination of Somatosensory and Viscerosensory Relay Neurons in the Hindbrain
}

\author{
Martin A. Sieber, ${ }^{1 *}$ Robert Storm, ${ }^{1 *}$ Margaret Martinez-de-la-Torre, ${ }^{2}$ Thomas Müller, ${ }^{1}$ Hagen Wende, ${ }^{1}$ Katja Reuter, \\ Elena Vasyutina, ${ }^{1}$ and Carmen Birchmeier ${ }^{1}$ \\ ${ }^{1}$ Department of Neuroscience, Max-Delbrück-Center for Molecular Medicine, 13125 Berlin, Germany, and 2Department of Human Anatomy and \\ Psychobiology, University of Murcia, 30100 Murcia, Spain
}

Distinct types of relay neurons in the hindbrain process somatosensory or viscerosensory information. How neurons choose between these two fates is unclear. We show here that the homeobox gene $L b x 1$ is essential for imposing a somatosensory fate on relay neurons in the hindbrain. In $L b x 1$ mutant mice, viscerosensory relay neurons are specified at the expense of somatosensory relay neurons. Thus Lbxl expression distinguishes between the somatosensory or viscerosensory fate of relay neurons.

Key words: neuronal specification; homeobox gene; nucleus of the solitary tract; spinal trigeminal nucleus; genetic lineage tracing; development

\section{Introduction}

Hindbrain neurons receive viscerosensory and somatosensory information from the periphery and integrate and relay this information. Neurons that receive somatosensory information of the face locate to the spinal trigeminal nucleus $(\mathrm{SpV})$, whereas the major relay station for visceral sensory information is the nucleus of the solitary tract (NTS) (Blessing, 1997; Qian et al., 2002). The homeodomain transcription factors Tlx3 and Lmx1b are expressed by developing sensory relay neurons of the NTS and SpV, indicating that these two neuronal types are ontogenetically related (Qian et al., 2002). An important factor that distinguishes the two neuronal subtypes is the paired-like homeodomain factor Phox $2 \mathrm{~b}$, which is expressed in viscerosensory but not somatosensory relay neurons (Dauger et al., 2003).

Neurons that relay viscerosensory and somatosensory information arise in a broad dorsal domain of the developing hindbrain denoted as the alar plate. Phox $2 b$ is a key factor for the specification of the viscerosensory neurons of the NTS, which do not develop in Phox2b mutant mice (Pattyn et al., 1999, 2000; Brunet and Pattyn, 2002). Phox $2 \mathrm{~b}$ is expressed in developing and mature NTS neurons, and Phox $2 \mathrm{~b}$ is therefore a useful marker for

Received May 11, 2005; revised March 8, 2007; accepted March 9, 2007.

C.B. was supported by grants from the Deutsche Forschungsgemeinschaft and the Bundesministerium für Bildung und Forschung. We thank Elvira Rohde for help with ES cell culture, Karin Gottschling and Jaqueline Andratscke for expert technical assistance, and Alistair Garratt, Walter Birchmeier, and Stefan Britsch for critically reading this manuscript. We gratefully acknowledge Luis Puelles for valuable advice and discussion and Silvia Arber for providing the Tau ${ }^{L a C Z}$ indicator mouse strain. We also thank Jean-Francois Brunet, Christo Goridis, David Anderson, Jane Johnson, Sandra Rebelo, Eric Turner, and Tom Jessell for gifts of antibodies and CDNA probes.

${ }^{*} M . A . S$. and R.S. contributed equally to this work.

Correspondence should be addressed to Carmen Birchmeier, Department of Neuroscience, Max-Delbrück-Center for Molecular Medicine, Robert Rössle Strasse 10, 13125 Berlin, Germany. E-mail: cbirch@mdc-berlin.de.

M. A. Sieber's present address: Bayer Schering Pharma AG, Müllerstrasse 170-178, 13353 Berlin, Germany.

DOI:10.1523/JNEUROSCI.0717-07.2007

Copyright $\odot 2007$ Society for Neuroscience $\quad$ 0270-6474/07/274902-08\$15.00/0 following prospective NTS neurons during their development and migration. The Phox $2 \mathrm{~b}+$ viscerosensory neurons express, in addition, Tlx3 and Lmx1b and are known to require Tlx3 for their development (Qian et al., 2001, 2002; Cheng et al., 2004). These neurons arise from a Mash1+ progenitor domain and are generated delayed and at reduced numbers in Mash1 mutant mice (Pattyn et al., 2006). Somatosensory neurons of the SpV also arise from the Mash1+ progenitor domain and express Tlx3 as well as Lmx1b, but not Phox2b (Qian et al., 2002).

Extensive cell migration and complex morphogenesis in the developing hindbrain can make it difficult to follow the development of hindbrain neurons. Recent progress in genetic fate mapping has helped to overcome this impediment (Landsberg et al., 2005; Machold and Fishell, 2005; Wang et al., 2005; Farago et al., 2006). For instance, genetic fate mapping was used to define the neuronal subtypes that arise from the dorsal edge of the developing hindbrain and the rhombic lip and give rise to the cerebellum and precerebellar nuclei (Landsberg et al., 2005; Machold and Fishell, 2005; Wang et al., 2005). The homeodomain factor Lbx1 is expressed in postmitotic neurons of the hindbrain that arise in a broad domain ventrally of the rhombic lip; we denote Lbx1+ neurons as "class B neurons." Here we use genetic fate mapping to define the derivatives of class B neurons in the medulla oblongata. The derivatives of class B neurons settle predominately in the dorsal and lateral medulla, and, in particular, they generate the somatosensory relay neurons of the SpV. In Lbxl mutant mice, $\mathrm{SpV}$ neurons are nor correctly specified, resulting in the absence of the $\mathrm{SpV}$. At their expense, mis-specified neurons arise that express Phox2b, migrate to the dorsal medulla, and contribute to the NTS. Consequently, the area occupied by Phox $2 \mathrm{~b}+$ neurons in the dorsal hindbrain is enlarged considerably. Our analysis allowed us to define changes in neuronal fates associated with the loss of $L b x 1$, and it shows that $L b x 1$ is an important 

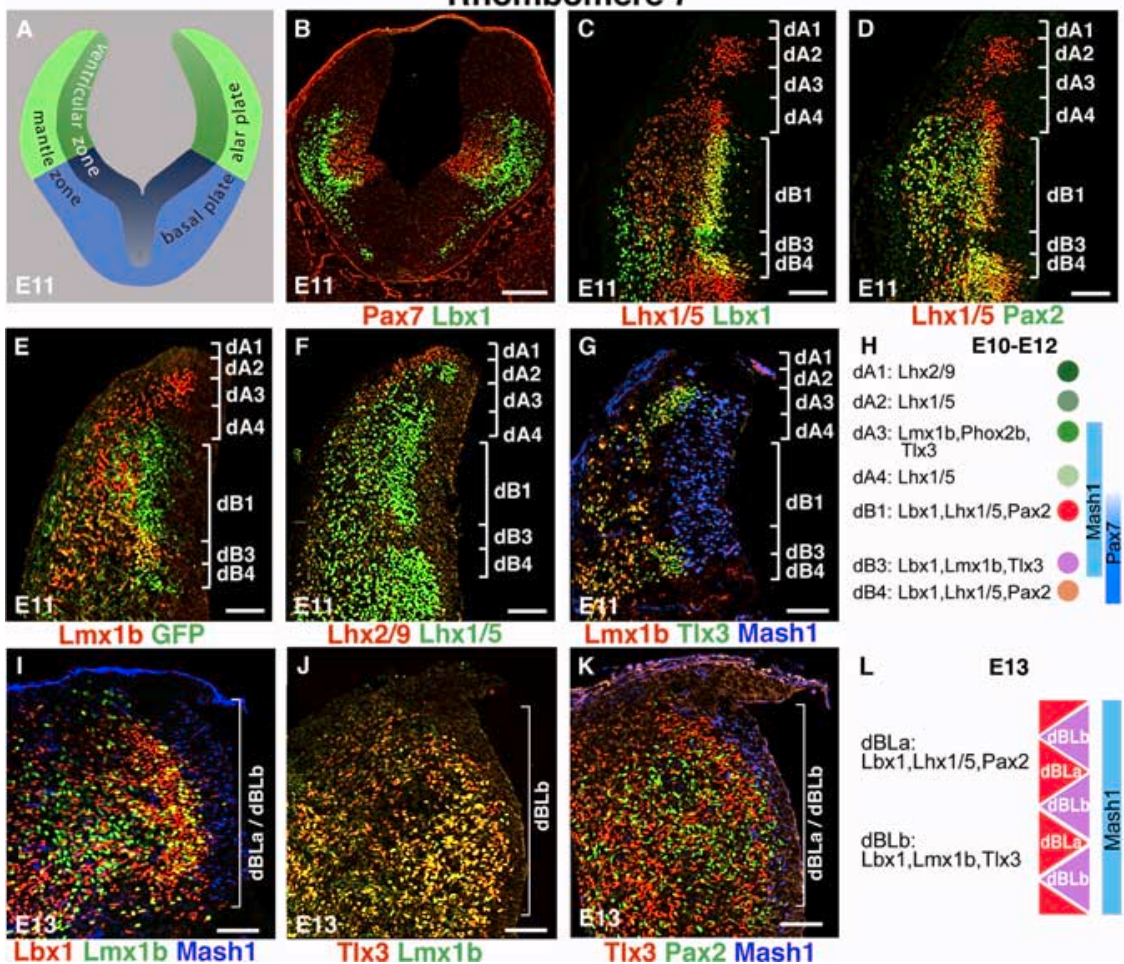

dA4: $\operatorname{Lh} \times 1 / 5$

dB1: Lbx1, Lhx1/5,Pax2

dB3: Lbx1,Lmx1b, Tlx 3 : dB4: Lbx1, Lhx1/5,Pax2
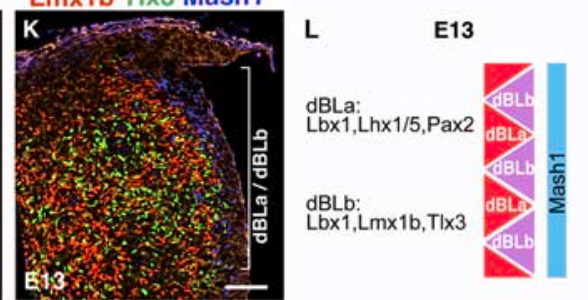

Tlx3 Pax2 Mash1

Figure 1. Characterization of neuronal subtypes generated in the alar plate of rhombomere 7. A, Schematic section through an E11 mouse hindbrain at the level of rhombomere 7. Alar and basal plates are indicated; dark colors denote the ventricular zone occupied by neuronal progenitors, and light colors indicate the mantle zone occupied by postmitotic neurons. Immunohistological analysis was performed on $\mathbf{L b x} 1^{\mathrm{GFP}} /+$ mice at E11 $(\boldsymbol{B}-\mathbf{G})$ and E13 $(\boldsymbol{I}-\boldsymbol{K})$. $\boldsymbol{B}$, Anti-Lbx1 (green) and anti-Pax7 (red) antibodies reveal that the $L b x 1+$ neurons appear lateral to the Pax7+ progenitor domain. $\mathbf{C}-\mathbf{G}$, Immunohistological analyses using the indicated antibodies at E11 define three Lbx1 + neuronal subtypes denoted as dB1, dB3, and dB4 that emerge in the ventral alar plate; brackets indicate the positions along the dorsoventral axis at which these neuronal subtypes emerge. In addition, four Lbx1 - dorsal neuronal subtypes denoted as $\mathrm{dA1}$ - $\mathrm{dA} 4$ emerge in the dorsal alar plate; brackets indicate the positions along the dorsoventral axis at which these neuronal subtypes emerge. $\mathbf{G}, \mathrm{dA} 3$ neurons correspond to the most dorsal neuronal subtype that emerges from the dorsal Mash1+ progenitor domain; $\mathrm{dB} 3$ is the most ventral neuronal subtype that emerges from Mash1+ progenitors. $\boldsymbol{H}$, Summary of early-born neuronal subtypes and of the transcription factors they express. I- $\boldsymbol{K}$, Immunohistological analyses using the indicated antibodies at E13 define two Lbx1 + neuronal subtypes that emerge from a broad domain in the alar plate denoted as $\mathrm{dBL}$ a and $\mathrm{dBLb}$; both emerge from a Mash1 progenitor domain in a salt-and-pepper pattern. $L$, Summary of late-born Lbx1+ neuronal subtypes and of the transcription factors these neuronal types express. Scale bars: $\boldsymbol{B}, 200 \mu \mathrm{m} ; \boldsymbol{C}-\mathbf{G}, 100 \mu \mathrm{m} ; \boldsymbol{I}-\boldsymbol{K}, 100 \mu \mathrm{m}$.

determinant in the differentiation of somatosensory neurons in the hindbrain.

\section{Materials and Methods}

Mouse strains. The Lbxl ${ }^{\mathrm{CreNeo}}$ mutant allele was generated by homologous recombination in embryonic stem (ES) cells; in this allele, a Cre cDNA as well as a neomycin cassette replace the first exon of $L b x 1$. Mutant ES cells were used to generate the corresponding mutant mouse strain. The neomycin cassette was removed by crossing mice carrying the $\mathrm{Lb} \times 1^{\mathrm{CreNeo}}$ allele with FLPe deleter mice (Farley et al., 2000; Rodriguez et al., 2000). The $L b x 1^{G F P}$ allele has been described previously (Vasyutina et al., 2005). Tau ${ }^{\text {LacZ }}$ (lox-STOP-lox-mGFP-IRES-NLS-LacZ-pA integrated into the second exon of the Tau locus) reporter mice were kindly provided by S. Arber (University of Basel, Basel, Switzerland) (Hippenmeyer et al., 2005). To perform genetic lineage tracing in a heterozygous and homozygous Lbxl mutant background, $L b \times 1^{C r e} /+$; Tau ${ }^{\mathrm{LacZ}}$ and $L b \times 1^{\mathrm{Cre}}$ / $\mathrm{Lbxl}^{\text {GFP }}$; Tau ${ }^{\mathrm{LacZ}}$ mice were analyzed, respectively.

Anatomy. We identified the different rhombomeric units by using morphological landmarks such as hindbrain nuclei and exit points of cranial nerves. We defined rhombomere 7 as the segment caudal to the facial nucleus and rostral to the fifth somite. Rhombomeres 4-6 were defined as the segments caudal to the pons, including the facial nucleus; rhombomeres 2 and 3 were defined as the segments of the pons (Marin and Puelles, 1995; Cambronero and Puelles, 2000).
Immunohistology, BrdU labeling, and microscopy. We used various antibodies to define neuronal subtypes that are born in the dorsal hindbrain. The expression of the corresponding genes and the changes in neuronal specification in $L b x 1$ mutant mice were verified by in situ hybridization. Immunohistology was essentially performed as described (Müller et al., 2002). In brief, embryos were fixed with $4 \%$ paraformaldehyde in $0.1 \mathrm{~m}$ sodium phosphate buffer, pH 7.4, equilibrated in 30\% sucrose, and embedded into OCT compound (Sakura Finetek, Torrance, CA) before cryosectioning. The following antibodies were used: guinea pig anti-Tlx3; guinea pig anti-Lbx1 (Müller et al. 2002); rabbit anti-Prxxl1 (kindly provided by $S$ Rebelo, University of Oporto, Porto, Portugal); goat anti- $\beta$-galactosidase (Biogenesis, Poole, UK), goat anti-green fluorescent protein (GFP) (Abcam, Cambridge, UK); rabbit anti-tyrosine hydroxylase (TH) (Pel-Freeze, Rogers, AZ); mouse anti-Mash1 (BD Biosciences PharMingen, Franklin Lakes, NJ); rabbit antiPhox2b (Christo Goridis and Jean-Francois Brunet, Ecole Normale Superieure, Paris, France); mouse anti-Neurofilament 68 (Sigma, Munich, Germany); rabbit anti-Pax2 (Zymed, San Francisco, CA); and monoclonal antiLhx1/5 (Developmental Studies Hybridoma Bank, Iowa City, IA). Guinea pig anti-Lmxlb and rabbit anti-Lhx2/9 were obtained from Tom Jessell and collaborators (Columbia University, New York, NY). Cyanine 2 (Cy2)-, Cy3-, or Cy5-conjugated donkey-antibodies (Dianova, Hamburg, Germany) were used for the detection of primary antibodies. For bromodeoxyuridine (BrdU) labeling experiments, BrdU (Sigma; $75 \mu \mathrm{g} / \mathrm{g}$ body weight) was injected intraperitoneally. Incorporated BrdU was detected with mouse (Sigma) or rat antiBrdU antibodies (Oxford Biotechnology, Oxfordshire, UK). Fluorescence was visualized with an LSM 5 PASCAL laser scanning microscope (Carl Zeiss, Jena, Germany), with PASCAL software (version 3.2). Pictures with a resolution of $512 \times 512$ or $1024 \times 1024$ pixels were taken with either a $10 \times$ Plan-Neofluar or a $20 \times$ Plan-Apochromat lens. For the display of larger sections, single pictures were taken with identical microscope settings and subsequently merged by using Adobe Photoshop.

\section{Results}

\section{Neuronal subtypes generated in the alar plate of} the hindbrain

The neural expression of the murine homeobox gene $L b x 1$ is first detected around embryonic day 10 (E10) and can be observed in rhombomeres 2-7 and at all axial levels of the spinal cord (Jagla et al., 1995; Schubert et al., 2001; Gross et al., 2002; Müller et al., 2002). We used anti-Lbx1 antibodies and $L b x 1^{\text {GFP }}$ heterozygous mice combined with anti-GFP antibodies to characterize Lbx1+ cells in transverse sections of the hindbrain. Lbx1+ (or GFP+) cells of heterozygous $L b \times 1^{\mathrm{GFP}} /+$ mice lie lateral to the Pax7+ progenitor domain in the ventral alar plate of the hindbrain (Fig. $1 A, B)$. BrdU labeling and the position of the Lbx $1+$ cells indicate that they correspond to postmitotic neurons (Fig. $1 B$ ) (data not shown). Using the nomenclature of the spinal cord, we denote the hindbrain Lbx1 + neurons that arise in the ventral part of the alar plate as class B dorsal neurons (cf. Müller et al., 2002). In 
addition, Lbx 1 - neurons arise dorsal to class B neurons in the alar plate of the hindbrain, and we denote these as class A.

Two phases of neurogenesis can be distinguished in the alar plate of rhombomere 7: an early (E10-E12) and a late (E12.5E15) phase. By using a panel of antibodies directed against transcription factors, we defined neuronal subtypes that arise in the two phases of neurogenesis. During the early phase, three Lbx1+ class B neuronal subtypes can be defined (Fig. $1 C-F$ ). The most ventrally emerging subtype, dB4, coexpresses Lbx1, Lhx1/5, and Pax2 (Fig. 1C,D). Dorsally abutting, dB3 neurons arise that express Lbx1, Lmx1b, and Tlx3 (Fig. 1C,E,G). The most dorsally arising class B neurons, $\mathrm{dB} 1$, express Lbx1, Lhx1/5, and Pax2 (Fig. $1 C-E)$. $\mathrm{dB} 3$ and $\mathrm{dB} 1$ neurons are lateral to a progenitor domain that expresses Mash1, indicating that they arise from the Mash1+ domain (Fig. 1G) (for a summary of the early arising dorsal neuronal subtypes in rhombomere 7, see Fig. $1 H$ ). In addition, four class A neuronal subtypes, dA1-4, are generated during the early phase in the dorsal alar plate of rhombomere 7 (Fig. $1 C-H$ ). One of these, the dA3 subtype, coexpresses Phox $2 b$, Tlx3, and Lmx1b (Fig. 1 and supplemental Fig. 1, available at www.jneurosci.org as supplemental material). dA3 neurons correspond thus to the developing viscerosensory neurons destined to form the NTS (Qian et al., 2001; Dauger et al., 2003). During the late neurogenic phase (E13), the domain that generates Lbx1 + neurons has expanded dorsally, and Lbx1 + neurons are observed lateral to the entire Mash1+ progenitor domain (Fig. $1 I-K$ ). All neurons that arise in this domain express Lbx1. Two late Lbx1+ neuronal subtypes, defined by the expression of either Lhx1/5/Pax2 or Lmx1b/Tlx3, appear to arise in a salt-and-pepper pattern. We denote these two late Lbx1+ neuronal subtypes as dBLa and dBLb (Fig. $1 I-L$ ).

In addition, we also characterized the neuronal types that emerge in the alar plate of rhombomeres 2-6. By using a panel of antibodies directed against Lbx1 (GFP), Lhx1/5, Lhx2/9, Pax2, Phox2b, Lmx1b, and Tlx3, we observed no obvious change in the neuronal subtypes emerging during early and late neurogenic phases in rhombomeres 2-6. Thus, the pronounced differences between neuronal subtypes appearing during early and late phases are specific for rhombomere 7 . We identified distinct numbers of neuronal subtypes of class A and class B in rhombomeres 2-6; however, two units could be defined, rhombomeres $4-6$ and rhombomeres $2-3$, in which dorsal neuronal subtypes with similar molecular characteristics are generated (Fig. 2A-D and supplemental Figs. 2 and 3, available at www. jneurosci.org as supplemental material).

\section{Derivatives of class B neurons in rhombomere 7}

Genetic fate mapping was used to define the derivatives of class B neurons. For this, we generated mice that carry an $L b \times 1^{C r e}$ allele by homologous recombination in ES cells (Fig. $3 A, B$ ). In the $L b \times 1^{C r e}$ allele, Cre sequences replace exon 1 of $L b \times 1$. A neomycin selection cassette (Neo) surrounded by two FRT (FLPerecombination target) sites was also inserted and removed subsequently (Fig. 3A) (see Material and Methods for additional details). Analysis of mice that carry the $L b \times 1^{\mathrm{Cre}}$ allele at E11 and E12.5 revealed that Cre expression was confined to Lbx1+ neurons (Fig. $3 C, D$ ). The $L b \times 1^{C r e}$ allele was used in mice to remove a DNA cassette that stops translation of a LacZ reporter (Tau ${ }^{\text {LacZ }}$ mice) (Hippenmeyer et al., 2005). The recombined LacZ gene is subsequently inherited, and the $L a c Z$ protein product, $\beta$-galactosidase, is detectable in cells that express Cre as well as in their derivatives. Derivatives of class B neurons identified by $\beta$-galactosidase expression in $L b \times 1^{\mathrm{Cre}} /+$; Tau ${ }^{\text {LacZ }}$ mice settled predominantly in the dorsal and lateral portions of the medulla
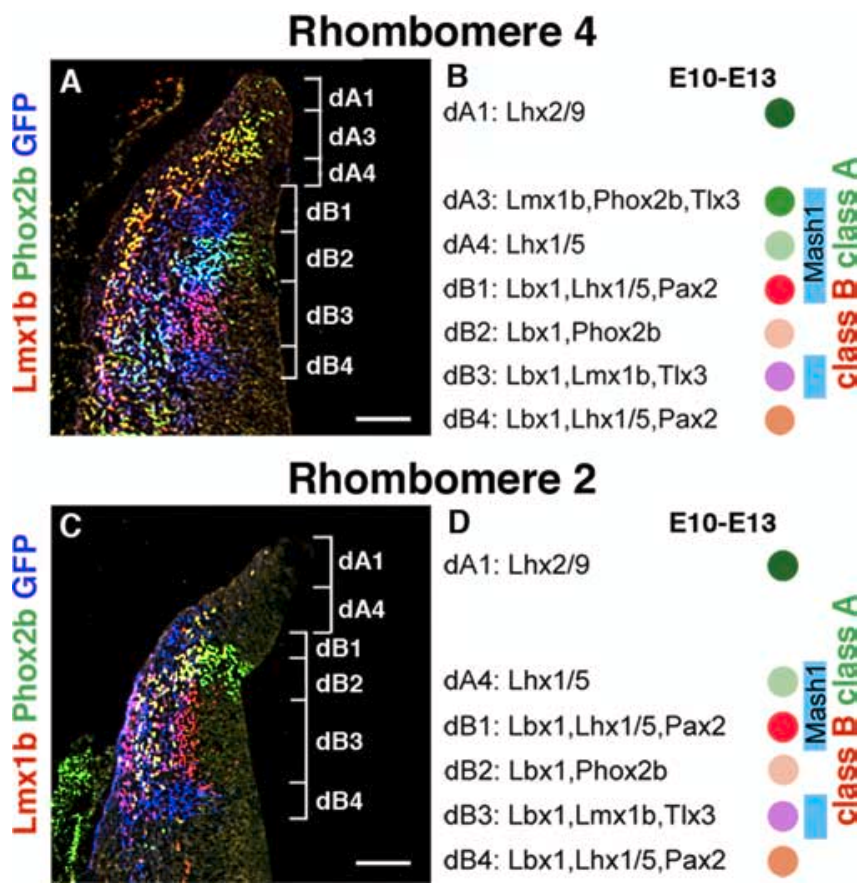

Figure 2. Characterization of neuronal subtypes generated in the alar plate of rhombomeres 2-6. A, Immunohistological analysis of rhombomere 4 of $L b x 1^{\mathrm{GFP}} /+$ mice at $\mathrm{E} 11$ with antibodies against Lmx1b (red), Phox2b (green), and GFP (blue) revealed three class $A$ and four class $B$ neuronal subtypes. $\boldsymbol{B}$, Summary of the neuronal subtypes emerging in rhombomeres 4-6. Note that immunohistological analyses using additional antibodies that either are displayed in supplemental Figures 2 and 3 (available at www.jneurosci.org as supplemental material) or not shown were used to define neuronal subtypes in rhombomeres $4-6$. C, Immunohistological analysis of rhombomere 2 of $L b \times 1^{\mathrm{GPP}} /+$ mice at E11 using antibodies against $L \mathrm{Lm} 1 \mathrm{~b}$ (red), Phox 2b (green), and GFP (blue). Brackets indicate the positions along the dorsoventral axis at which the neuronal subtypes emerge. $\boldsymbol{D}$, Summary of the neuronal subtypes emerging in rhombomeres 2 and 3 . Note that immunohistological analyses using additional antibodies that either are displayed in supplemental Figures 2 and 3 (available at www.jneurosci.org as supplemental material) or not shown were used to define neuronal subtypes in rhombomeres 2 and 3 . Scale bars, $100 \mu \mathrm{m}$.

oblongata (Fig. $3 E$ ). In particular, Lmxlb + neurons in the $\mathrm{SpV}$ derive from class B neurons (Fig. $3 F$ ). In the NTS, neurons that derive from the class $B$ lineage are also observed, but these do not express Phox $2 \mathrm{~b}+$ (Fig. 3G). Many $\beta$-galactosidase + neurons in the NTS coexpressed Pax2; a more detailed characterization and the identification of the origin of Pax $2+$ neurons in the NTS will be reported elsewhere.

\section{Phox $2 b+$ viscerosensory neurons are generated in increased} numbers in Lbx 1 mutant mice

The future NTS neurons (dA3; Phox $2 \mathrm{~b}+/ \mathrm{Lmx} 1 \mathrm{~b}+/ \mathrm{Tlx} 3+)$ arise dorsally during the early neurogenic phase and migrate in a ventral direction (Fig. 4A,C) (Dauger et al., 2003). They settle at a position adjacent to vagal motoneurons, which also express Phox $2 b$ but are distinguishable from dA3/NTS neurons because they are negative for Lmxlb (Fig. $4 A, C$, arrowheads point to vagal motoneurons born in a ventral domain, and arrows point to dA3/NTS neurons) [for a detailed description of vagal motoneurons and dA3/NTS neurons, see also Dauger et al. (2003)]. In Lbx1 mutant mice, many ectopic neurons arose in the ventral alar plate that express Phox $2 \mathrm{~b}, \mathrm{Lmx} 1 \mathrm{~b}$, and Tlx3 and thus display the molecular identity of dA3 neurons (Fig. 4A-D) (data not shown). During the early neurogenic phase, these ectopic neurons were generated at the positions at which $\mathrm{dB} 3$ neurons are generated in normal development, and they arose at the expense of $\mathrm{dB} 3$ neu- 

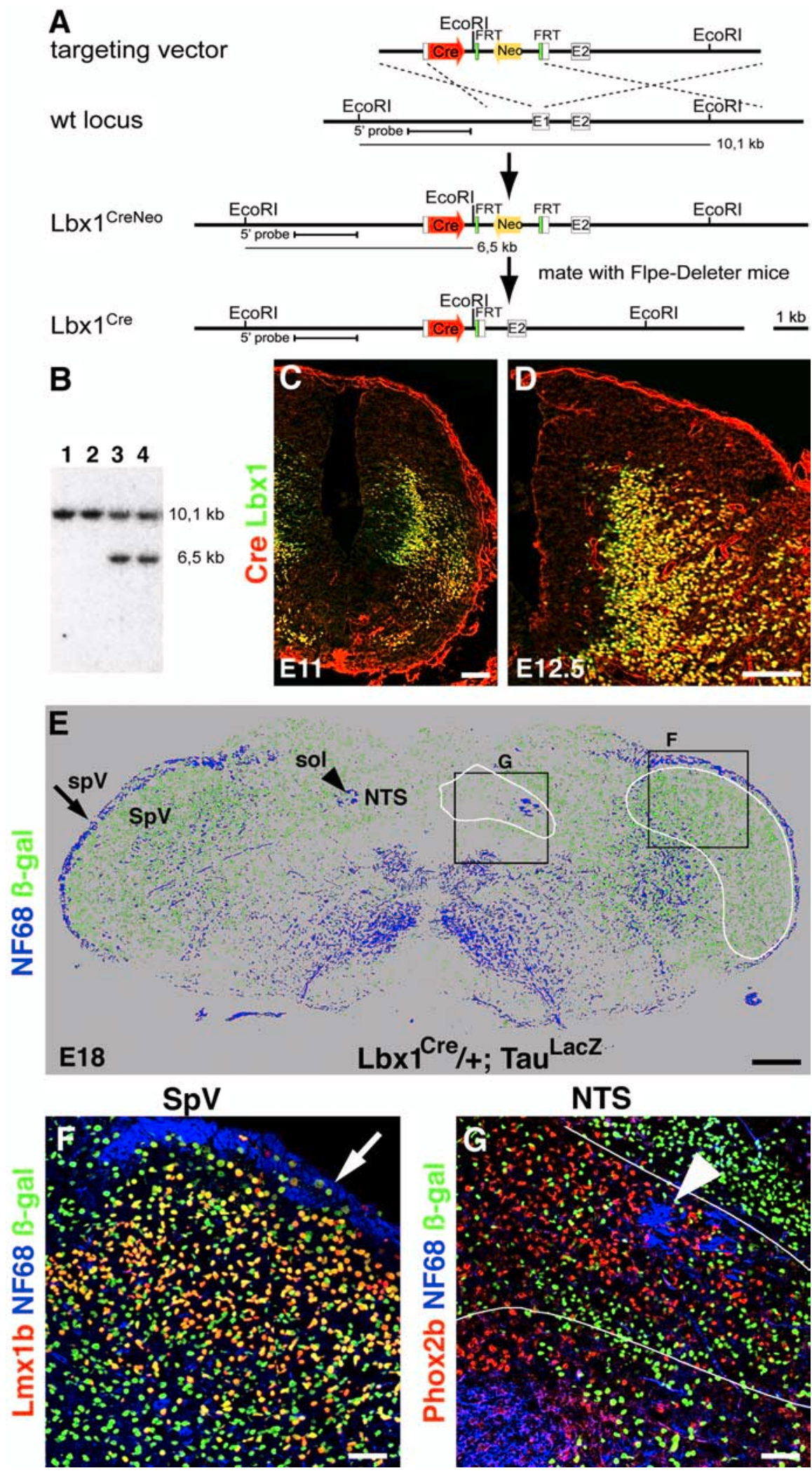

Figure 3. Genetic lineage tracing defines the derivatives of the class B neurons of rhombomere 7. $\boldsymbol{A}$, Strategy used to generate the $L b \times 1^{C r e N e o}$ allele by homologous recombination. Schematic representation of the targeting vector, the wild-type $L b \times 1$ locus, and the mutant $L b x 1$ alleles before $\left(L b \times 1^{C r e N e o}\right)$ and after $\left(L b \times 1^{C r e}\right)$ removal of the neomycin (Neo) cassette. The $L b \times 1$ gene has two exons (E1 and E2); the first was interrupted by the insertion of a Cre-FRT-Neo-FRT cassette. Indicated are (re (red) and FRT (green) sequences, the neomycin resistance cassette (yellow), the sequence ( $5^{\prime}$ probe) used for Southern analysis shown in $\boldsymbol{B}$, and EcoR restriction sites. $\boldsymbol{B}, \mathrm{ECORI}$-digested DNA of ES cell clones (lanes 1 and 2 , wild type; lanes 3 and $4, \mathrm{Lb} 1^{\mathrm{CreNeo}} /+$ ) hybridized with the sequence indicated in $\boldsymbol{A}$ ( $5^{\prime}$ probe). $\boldsymbol{C}, \boldsymbol{D}$, Immunohistological analyses of rhombomere 7 in $L b \times 1^{C r e} /+$ mice at E11 (C) and E12.5 (D), using anti-Cre (red) and anti-Lbx1 (green) antibodies. $\boldsymbol{E}$, Lineage tracing using $\mathrm{Lbx} 1^{\mathrm{Cre}} /+$; Tau ${ }^{\mathrm{LaCZ}}$ mice. Derivatives of the rons (Fig. $4 A, B$ ). In addition, ectopic Phox $2 \mathrm{~b}+/ \mathrm{Lmx} 1 \mathrm{~b}+/ \mathrm{Tlx} 3+$ neurons appeared to arise also during the late neurogenic phase (Fig. 4C,D) (data not shown). BrdU labeling experiments confirmed that these ectopic neurons were generated during the late phase, in contrast to the Phox $2 \mathrm{~b}+$ neurons (NTS neurons and vagal motoneurons) of control mice that arose exclusively during the early neurogenic phase (Fig. 4E,F). These ectopic late-born Phox $2 b+$ neurons were generated at the expense of dBLb neurons (Fig. $4 D)$. We conclude, therefore, that $\mathrm{dB} 3$ and $\mathrm{dBLb}$ neurons are mis-specified and denote the ectopic neurons that arose at their expense as $\mathrm{dB}^{*}$ and $\mathrm{dBLb}^{*}$ neurons. $\mathrm{dB}^{*}$ and $\mathrm{dBLb}^{\star}$ neurons display the molecular characteristics of $\mathrm{dA} 3$ neurons.

To follow the consequences of the appearance of $\mathrm{dB}^{*}$ and $\mathrm{dBLb}^{*}$ neurons, the medulla of $L b x 1$ mutant mice was analyzed at subsequent developmental stages (E14 and E18). At these stages, dA3/NTS neurons have attained their final position close to the solitary tract as a result of morphogenetic movements of the hindbrain (Fig. 5A, C,E) (Qian et al., 2001; Dauger et al., 2003). We observed a pronounced increase in the number of neurons that coexpressed Phox $2 \mathrm{~b}$ and Lmx1b in the Lbx 1 mutant compared with control mice (Fig. $5 A-F$, arrows). The supernumerary Phox $2 \mathrm{~b}+$ and Lmx1b + neurons assemble in a broad dorsal band. This indicates that mis-specified $\mathrm{dB}^{*} / \mathrm{dBLb}^{*}$ neurons not only assume the molecular identity of dA3 neurons, but they also settle like dA3 neurons in the dorsal medulla close to the solitary tract.

Lbx1 + class B neurons were identified by immunohistology with antibodies directed against $\beta$-galactosidase ( $\beta$-gal; green); sections of the medulla oblongata were counterstained with antibodies against neurofilament 68 (NF68; blue) to ease orientation. The solitary tract (sol; arrowhead), the spinal trigeminal tract (spV; arrow), the NTS, and the SpV are indicated. To improve the visibility of neurons, a false color was assigned to the background that appeared in black in the original photograph. Sections in $\boldsymbol{F}$ and $\mathbf{G}$ show the $\mathrm{SpV}$ and NTS, respectively, and correspond to the boxed areas shown in $E$. F, Analysis of the $S p V$ of $L b x 7^{\text {Cre }} /+; \operatorname{Tau}^{\operatorname{LacZ}}$ mice by using antibodies directed against Lmx1b (red), $\beta$-galactosidase (green), and neurofilament (blue). Note that most of the Lmx $1 \mathrm{~b}+$ neurons coexpress $\beta$-galactosidase. $\mathbf{G}$, Analysis of the NTS of $L b x 7^{\mathrm{Cre}} /+$; Tau ${ }^{\mathrm{LaCZ}}$ mice by using antibodies directed against Phox2b (red), $\beta$-galactosidase (green), and neurofilament (blue). Note that most of the Phox $2 b+$ neurons do not express $\beta$-galactosidase. Scale bars: $\boldsymbol{C}, \boldsymbol{D}, 100$ $\mu \mathrm{m} ; \boldsymbol{E}, 200 \mu \mathrm{m} ; \boldsymbol{F}, \boldsymbol{G}, 50 \mu \mathrm{m}$. 

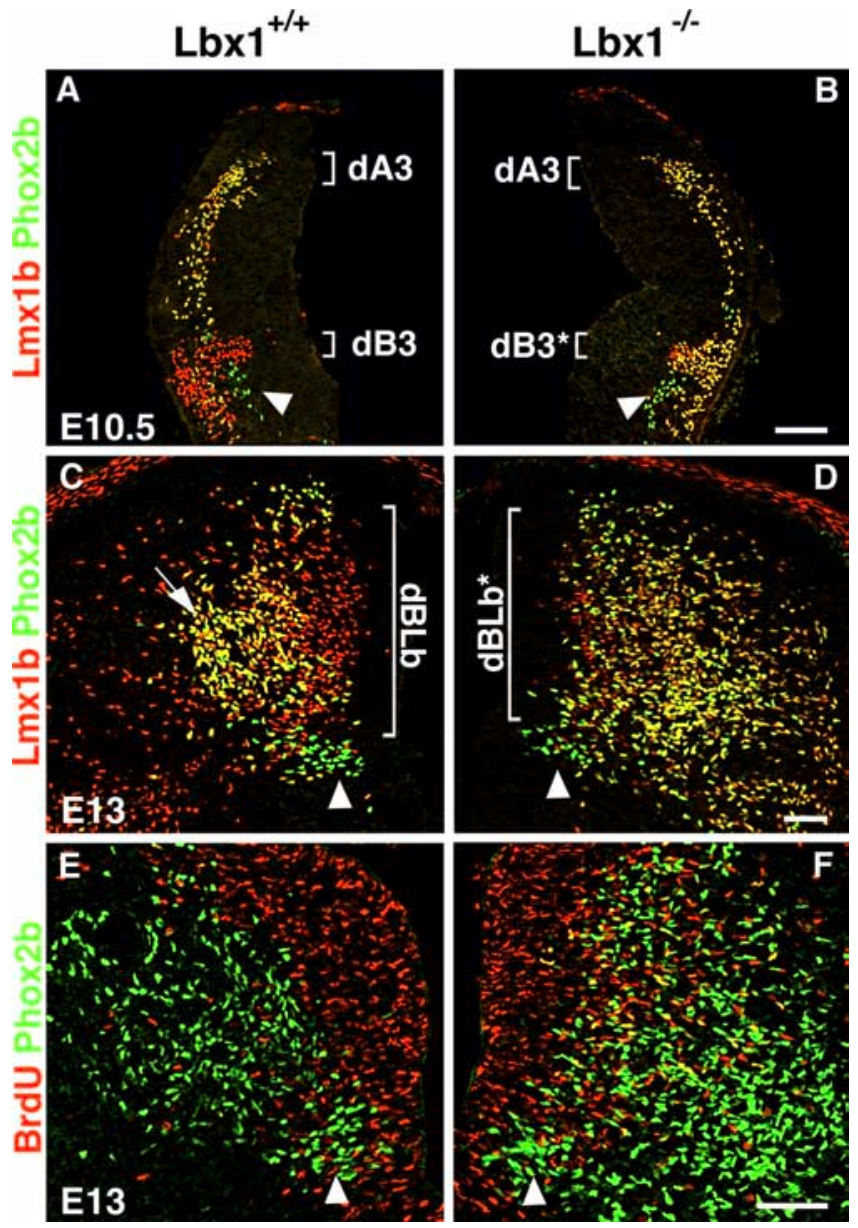

Figure 4. $L b x 1$ is essential for the correct specification of $d B 3$ and $d B L b$ neurons in rhombomere 7. Immunohistological analyses are shown of the alar plate of rhombomere 7 of control $(\boldsymbol{A}, \boldsymbol{C}, \boldsymbol{E})$ and $L b x 1$ mutant $(\boldsymbol{B}, \boldsymbol{D}, \boldsymbol{F})$ mice at E10.5 $(\boldsymbol{A}, \boldsymbol{B})$ and E13 $(\boldsymbol{C}-\boldsymbol{F})$. Arrowheads point toward vagal motoneurons. $\boldsymbol{A}, \boldsymbol{B}$, Immunohistological analysis using anti-Phox2b (green) and anti$\mathrm{Lmx} 1 \mathrm{~b}$ (red) antibodies. In control embryos at E10.5, dA3 neurons coexpress Phox $2 \mathrm{~b}$ and $\mathrm{Lm} \times 1 \mathrm{~b}$ and appear yellow (indicated by bracket); vagal motoneurons (arrowhead) are Phox $2 b+$ and $\mathrm{Lmx} 1 \mathrm{~b}$ - and appear green; and dB3 neurons are Lmx1b + and Phox $2 b$ - and appear red. In Lbx 1 mutant mice, Phox $2 \mathrm{~b}$ and Lmx $1 \mathrm{~b}$ are coexpressed in mis-specified dB3* and in $\mathrm{dA} 3$ neurons. $\boldsymbol{C}, \boldsymbol{D}$, Immunohistological analysis using anti-Phox2b (green) and anti-Lmx1b (red) antibodies. In control embryos at E13, dA3 neurons (Phox2b+/Lmx1b +; these neurons appear yellow and are indicated by an arrow) are positioned dorsal to the vagal motoneurons (Phox2b + and appearing green; indicated by arrowhead). Lmx1b + dBLb neurons (red, indicated by a bracket) emerge at this stage from a broad domain of the alar plate. In Lbx1 mutant mice, mis-specified dBLb neurons (denoted as dBL $b^{*}$ ) coexpress Phox $2 b$ and $L m \times 1 b$ and appear yellow. $\boldsymbol{E}, \boldsymbol{F}$, Pulse- chase experiment using BrdU. BrdU was injected at E12.5, and analysis was performed at 13 with antibodies directed against Phox2b + (green) and BrdU (red). In control mice, BrdU was not incorporated into Phox2b + neurons. In Lbx1 mutant mice, a number of BrdU +/Phox $2 b+$ neurons appear in a broad domain of the alar plate. Scale bars: $A, B, 100$ $\mu \mathrm{m} ; \boldsymbol{C}-\boldsymbol{F}, 50 \mu \mathrm{m}$.

The trigeminal somatosensory relay nucleus is absent in $L b x 1$ mutant mice

$\mathrm{dBLb}$ neurons, which arise in the alar plate during the late neurogenic phase, have settled by E14 in the lateral medulla (Fig. 5A, asterisk). By E18, the $\mathrm{SpV}$ has formed at this site and continues to contain Lmx1b+ and Tlx3+ neurons (Fig. 5C and supplemental Fig. $4 A$, available at www.jneurosci.org as supplemental material). In $L b x 1$ mutant mice, the Lmx1b+ and Tlx3 + neurons of the SpV cannot be discerned at E14 and E18 (Fig. 5B,D and supplemental Fig. $4 B$, available at www.jneurosci.org as supplemental material). The SpV of control mice at E18 contains many
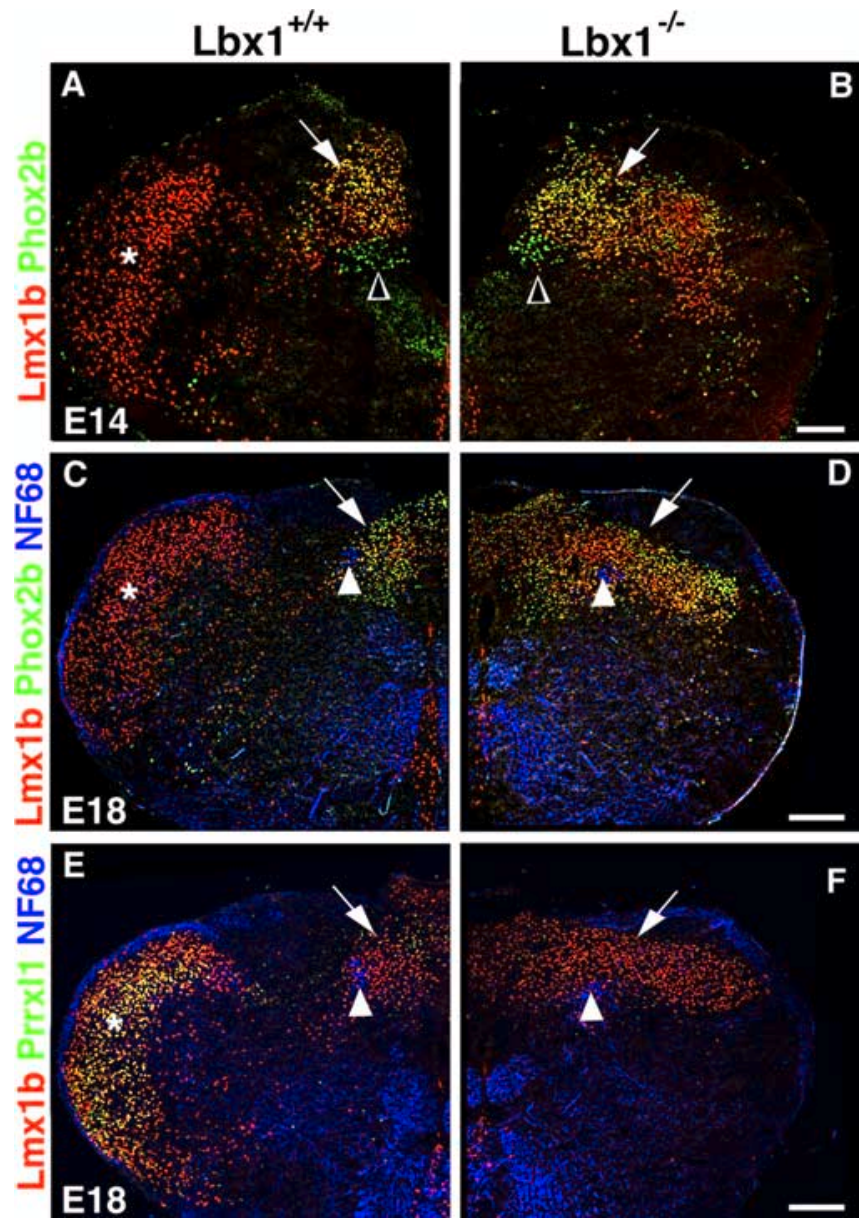

Figure 5. Appearance of the SpV and NTS in Lbx1 mutant mice. Immunohistological analyses are shown of the medulla of control $(\boldsymbol{A}, \boldsymbol{C}, \boldsymbol{E})$ and $L b x 1$ mutant $(\boldsymbol{B}, \boldsymbol{D}, \boldsymbol{F})$ mice at E14 $(\boldsymbol{A}, \boldsymbol{B})$ and E18 $(\boldsymbol{C}-\boldsymbol{F}) . \boldsymbol{A}, \boldsymbol{B}$, Immunohistological analysis using antibodies directed against anti-Phox2b (green) and anti-Lmx1b (red). In control mice, dA3 neurons (yellow, arrow) are positioned dorsal to vagal motoneurons (Phox2b +; green, open arrowhead) in the dorsomedial medulla where the NTS is forming. Lmx1b + neurons (red) form a vertical band in the lateral medulla where the SpV is forming (asterisk). In Lbx 1 mutant mice, increased numbers of neurons located in a broad dorsal band coexpress $L m \times 1 b+$ and Phox $2 b$, and the lateral band of $L m \times 1 b+$ neurons is absent. $\mathbf{C}, \mathbf{D}$, Immunohistological analysis using antibodies directed against $L m \times 1 b$ (red), Phox2b (green), and neurofilament (NF68, blue). The solitary tract (arrowhead) and the NTS (arrow) are indicated. In the lateral medulla of control mice, $\mathrm{Lmx} 1 \mathrm{~b}+$ neurons have settled in the SpV (asterisk). In $L b x 1$ mutant mice, a broad dorsal band of neurons that coexpress $L m x 1 b$ and Phox2b exists (yellow); Lmx1b + neurons (red) are not observed in the lateral medulla. $\boldsymbol{E}$, $\boldsymbol{F}$, Immunohistological analysis using antibodies directed against Lmx1b (red), Prrxl1 (green), and neurofilament (NF68, blue). In control mice, many neurons in the SpV (asterisk) coexpress Lmx $1 b$ and Prrxl1 (yellow). In Lbx1 mutant mice, neurons that coexpress Lmx1b and Prrxl1 are not observed in the lateral medulla. Scale bars: $A, B, 100 \mu \mathrm{m} ; \boldsymbol{C}-\boldsymbol{F}, 200 \mu \mathrm{m}$.

neurons that coexpressed Lmx1b and high levels of Prrxl1 (also known as Drg11), and such neurons were not present in the lateral medulla of the $L b x 1$ mutant mice (Fig. $5 E, F$ ); therefore, we conclude that the SpV does not form in $L b x 1$ mutant mice.

To assess this further, we performed fate-mapping experiments on Lbx1 heterozygous and homozygous mutant backgrounds with $L b \times 1^{C r e}$ mice. Cre expression was restricted to a similar domain in the ventral alar plate in heterozygous $\left(\mathrm{Lb} \times 1^{\mathrm{Cre}} /+\right.$; $\left.\mathrm{Tau}^{\mathrm{LacZ}}\right)$ and homozygous ( $\mathrm{Lb} \times 1^{\mathrm{Cre}} / \mathrm{Lb} \times 1^{\text {GFP }}$; $\mathrm{Tau}^{\text {LacZ }}$ ) mutant mice (data not shown). In heterozygous $L b \times 1$ mutant mice at E18, a large group of $\beta$-galactosidase + neurons located to the $\mathrm{SpV}$ in the lateral medulla, and this group was not discernable in the homozygous mutant (Fig. 6A, $B$ ). The absence 

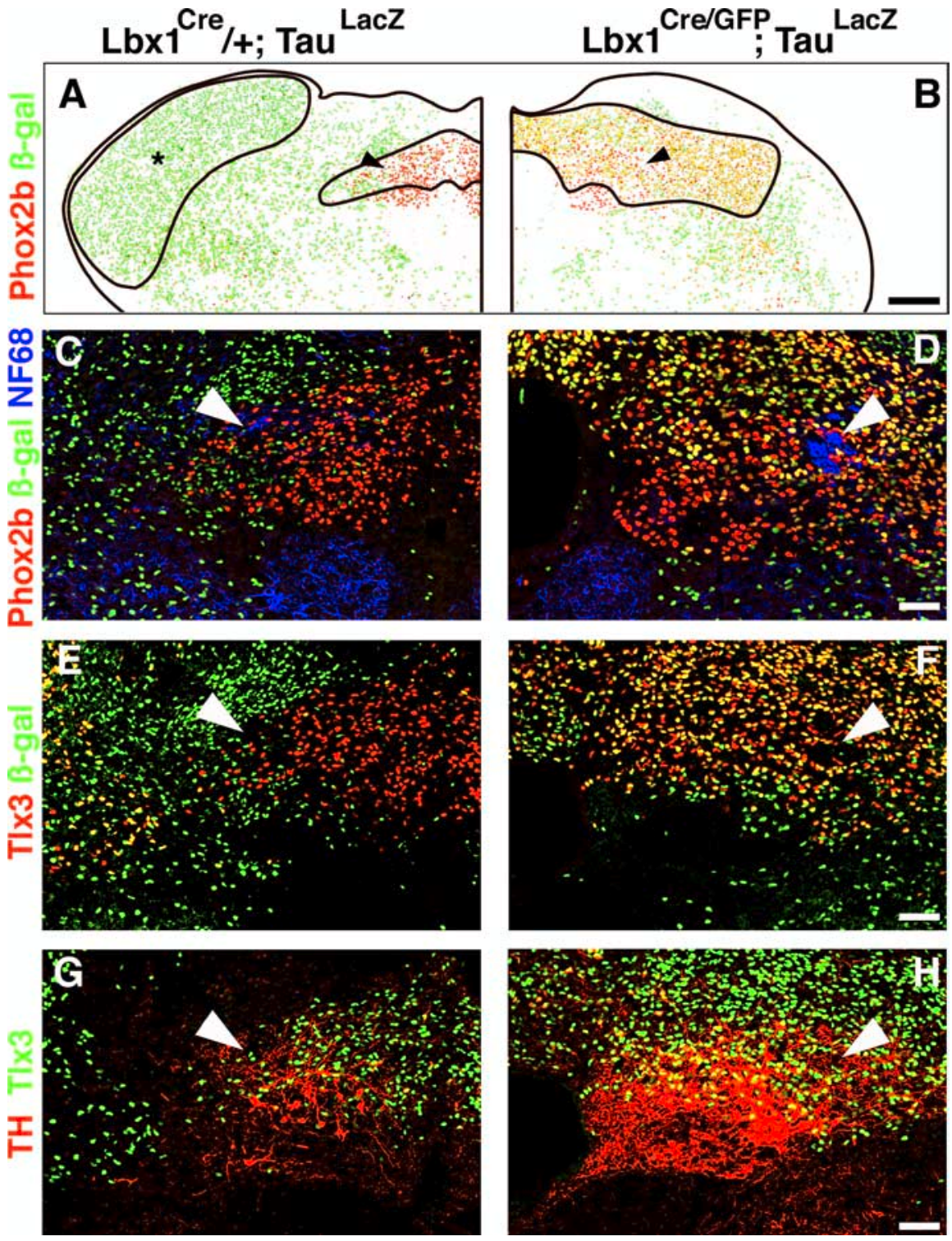

Figure 6. Genetic lineage tracing in $L b \times 1$ mutant mice. Analyses of neuronal fates in the caudal medulla of control $\left[L_{b x}\right]^{C r e} /+$; $\left.\operatorname{Tau}^{L a C Z}(\boldsymbol{A}, \boldsymbol{C}, \boldsymbol{E})\right]$ and $L b \times 1$ mutant $\left[\mathrm{Lbx} 7^{\mathrm{Cre}} / \mathrm{Lbx} 1^{G F P} ; \operatorname{Tau}^{\mathrm{LaCZ}}(\boldsymbol{B}, \boldsymbol{D}, \boldsymbol{F})\right]$ mice at E18. Lineage tracing was performed by the identification of $\beta$-galactosidase-expressing neurons. $A, B$, Immunohistological analysis of the dorsal medulla using antibodies directed against $\beta$-galactosidase ( $\beta$-gal, green) and Phox $2 \mathrm{~b}$ (red). To improve the visibility of neurons, a false color was assigned to the background that appeared in black in the original photograph; arrowheads point toward the solitary tract. In the control $(\boldsymbol{A})$, the SpV (asterisk) and the NTS are outlined. In the $L b x 1$ mutant $(\boldsymbol{B})$, the enlarged NTS occupied by neurons coexpressing $\beta$-galactosidase and Phox $2 b$ is outlined. $C-H$, Consecutive sections of the medulla of control and $L b x 1$ mutant mice. The area close to the solitary tract is shown, and the position of the solitary tract is indicated by arrowheads. $C, D$, Immunohistological analysis using antibodies directed against Phox2b (red), $\beta$-galactosidase (green), and neurofilament (NF68, blue). Note that Phox2b+ neurons do not coexpress $\beta$-galactosidase in control mice. Many neurons that coexpress $\beta$-galactosidase and Phox $2 \mathrm{~b}+$ appear in the $L b \times 1$ mutant. $\boldsymbol{E}, \boldsymbol{F}$, Immunohistological analysis using antibodies directed against TIx3 (red) and $\beta$-galactosidase (green). Note that TIx3 + neurons of the NTS do not coexpress $\beta$-galactosidase in control mice. In the $L b \times 1$ mutant, many TIx3 + neurons of the NTS coexpress $\beta$-galactosidase. $\boldsymbol{G}, \boldsymbol{H}$, Immunohistological analysis using antibodies directed against Tlx3 (green) and TH (red). Note the increase in TH staining in $L b x 1$ mutant mice. Scale bars: $A, B, 200 \mu \mathrm{m} ; \boldsymbol{C}-\boldsymbol{H}, 50 \mu \mathrm{m}$.

of this large group of neurons apparently causes the abnormal shape of the medulla of the mutant mice, i.e., the reduction of its width. This was accompanied by the appearance of supernumerary $\beta$-galactosidase + neurons in the dorsal medulla (Fig. $6 A, B$ ). Phox $2 \mathrm{~b}+$ or Tlx $3+$ neurons of the NTS did not express $\beta$-galactosidase in heterozygous mice (Fig. $6 A, C, E$ ). In contrast, many dorsal Phox $2 \mathrm{~b}+$ or $\mathrm{Tlx} 3+$ neurons expressed $\beta$-galactosidase in homozygous mutants (Fig. $6 B, D, F$ ). Some NTS neurons express TH in control mice; these correspond to neurons of the $\mathrm{A} 2 / \mathrm{C} 2$ noradrenergic center. The number of $\mathrm{TH}+$ neurons was increased in homozygous $L b x 1$ mutant mice (Fig. 6G,H). We conclude that mis-specified class $\mathrm{B}$ neurons generate the supernumerary Phox $2 \mathrm{~b}+$ neurons that assemble in the dorsal medulla.

\section{Discussion}

Expression of the homeodomain transcription factor Lbx1 marks a major class of neurons (class B) that emerges in the ventral alar plate of rhombomeres $2-7$ of the hindbrain. Our genetic fate mapping shows that derivatives of class $\mathrm{B}$ neurons generate the $\mathrm{SpV}$ nucleus that processes somatosensory information. The analysis of $L b x 1$ mutant mice revealed that the future SpV neurons are not correctly specified and that the SpV does not form. This is caused by a mis-specification of the neurons destined to generate the $\mathrm{SpV}$, and we provide evidence that the mis-specified neurons contribute instead to the NTS. Lbxl acts thus as a postmitotic determinant and controls the choice between the specification of somatosensory versus viscerosensory fates of relay neurons in the medulla oblongata.

\section{Lbxl and the specification of sensory relay neurons}

We used the expression of Lbx1 to distinguish two classes of neuronal subtypes born in the alar plate of rhombomeres 2-7 of the hindbrain: class $A(\mathrm{Lbx} 1-)$ and class B $(\operatorname{Lbx} 1+)$. For the further characterization of neuronal subtypes of the two classes, we used a panel of antibodies directed against the transcription factors Lhx1/5, Lhx2/9, Pax2, Phox2b, Lmx1b, and Tlx3. We found that neuronal subtypes differ among rhombomeres; however, three units exist that possess similar characteristics: rhombomeres 2 and 3, rhombomeres 4-6, and rhombomere 7 . Within these three units, identical sets of neuronal subtypes of class A and class B are born. The three units mature into three functional and anatomical compartments of the mature hindbrain: the pons, the anterior medulla oblongata, and the posterior medulla oblongata. The three units defined by the sets of emerging neuronal subtypes coincide with pro-rhombomeric units defined by early morphological analyses (Bergquist and Kallen, 1955; Vaage, 1969).

Genetic lineage tracing was used to characterize the further developmental potential of Lbx1+ class B neurons in the medulla. We observe that derivatives of class B neurons settle predominantly in the dorsolateral domain. In particular, the SpV derives from class B neurons; this nucleus relays somatosensory information received via the trigeminal nerve. At E18, many neu- 


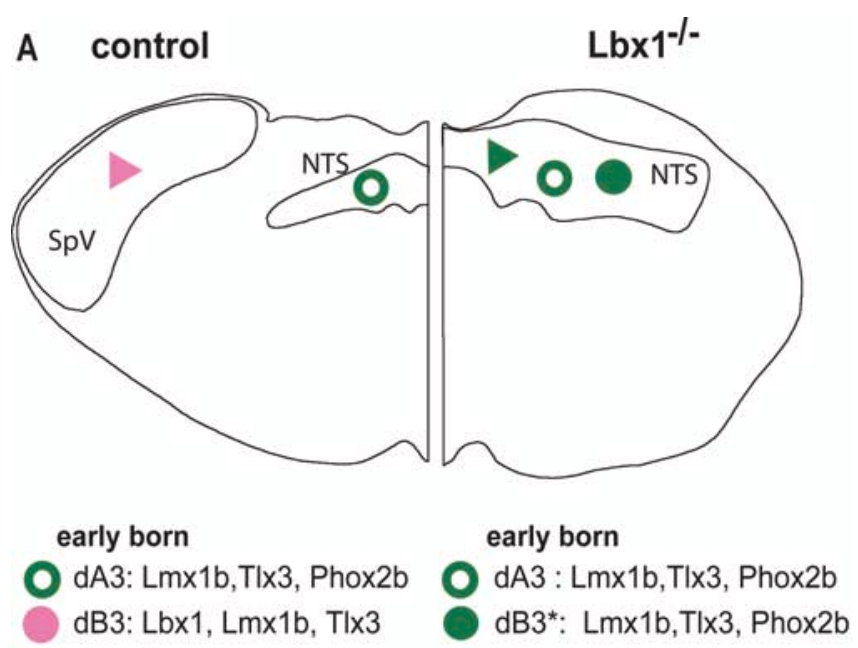

late born:

dBLb: Lbx1, Lmx1b,Tlx3

late born:

dBLb*: Lmx1b,TIx3,Phox2b

B

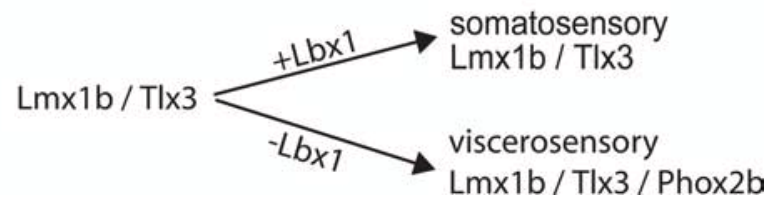

Figure 7. Summary of the changes in cell fate observed in Lbx1 mutant mice. $A$, Schematic drawing of the medulla oblongata of control (left) and $L b \times 1$ (right) mutant mice. In control mice, $d B L b$ neurons (pink triangles) that are born during late stages of neurogenesis generate the $\mathrm{SpV} ; \mathrm{dA} 3$ neurons (green open circles) are born at early stages of neurogenesis and generate the NTS. In $L b x 1$ mutant mice, $d B L b *$ (green triangles) and $d B 3^{*}$ (green filled circles) neurons are generated at the expense of $d B L b$ and $d B 3$ neurons. $d B L b^{*}$ neurons assume the molecular characteristics of $\mathrm{dA} 3$ neurons and contribute to the NTS; $\mathrm{dB} 3^{*}$ neurons also display molecular characteristics of $\mathrm{dA} 3$ neurons, and dB3* neurons might join the NTS. $B$, Lmx $1 \mathrm{~b}$ and TIX3 coexpression marks neurons that are destined to generate sensory relay neurons. In the presence of $\mathrm{Lbx} 1$, these neurons assume the fate of somatosensory relay neurons and generate the SpV. In the absence of $L b x 1$, these neurons assume the fate of viscerosensory relay neurons and contribute to the NTS.

rons of the SpV express Lmx1b and Tlx3, and frequently these neurons coexpress Lbx1 (Fig. 5C and supplemental Fig. 4, available at www.jneurosci.org as supplemental material) (data not shown). Birth-dating experiments indicate that the SpV is generated by neurons born after E12, i.e., during the late phase of neurogenesis (Altman and Bayer, 1980) (M. A. Sieber and R. Storm, unpublished observations). During the late phase of neurogenesis, one large neuronal population that expresses Lmxlb, Tlx3 and Lbx1 is generated that we denote as dBLb. These neurons appear to migrate laterally and were previously proposed to contribute to the SpV (Qian et al., 2002). We analyzed the generation of dBLb neurons in $L b x 1$ mutant mice and observed that these neurons are not correctly specified and that the $\mathrm{SpV}$ is not formed. This shows that $\mathrm{dBLb}$ neurons generate a major population of SpV neurons in normal development (Fig. 7).

In $L b x 1$ mutant mice, we observed that a neuronal subtype is born at the expense of $\mathrm{dBLb}$ that we denote as $\mathrm{dBLb}^{*}$. $\mathrm{dBLb}^{*}$ neurons arise during the late phase of neurogenesis and constitute a large neuronal population that expresses Phox $2 b, \mathrm{Lmx} 1 \mathrm{~b}$, and Tlx3. The molecular characteristics of these neurons thus resemble those of $\mathrm{dA} 3$ neurons, which are destined to form the NTS in normal development (Dauger et al., 2003). In Lbx1 mutant mice at E18, many supernumerary Phox $2 \mathrm{~b}+$ neurons assemble in a broad dorsal band near the solitary tract. Lineage tracing as well as BrdU labeling experiments show that many ectopic Phox2b neurons, which derive from cells that express Lbx1 in normal development, were born during a late neurogenic phase. A subgroup of neurons in the NTS that corresponds to the A2/C2 noradrenergic center expresses TH in control mice, and we observed an increased number of $\mathrm{TH}+$ neurons in $\mathrm{Lb} \times 1 \mathrm{mu}-$ tant mice. Together, these data indicate that the $\mathrm{dBLb}^{*}$ neurons do not only assume the molecular characteristics of dA3 neurons, but they also settle at the dorsal position at which $\mathrm{dA} 3$ neurons settle in normal development. The small population of $\mathrm{dB}^{*}$ neurons also displays dA3 characteristics in $L b \times 1$ mutant mice and might further contribute to the large group of ectopic Phox $2 b$ neurons that assemble dorsally. We conclude that Lbx1 is essential to specify dBLb neurons that, in normal development, generate somatosensory relay neurons of the SpV. In $L b x 1$ mutant mice, they assume the molecular characteristics of neurons that generate a viscerosensory relay nucleus, the NTS (Fig. 7).

\section{Molecular mechanisms of neuronal specification in the spinal cord and hindbrain}

The molecular mechanisms that control the specification of different neuronal subtypes along the dorsoventral axis have been analyzed extensively in the spinal cord (for reviews, see Lee and Jessell, 1999; Jessell, 2000; Briscoe and Ericson, 2001; Caspary and Anderson, 2003). Frequently, neuronal subtypes with similar molecular characteristics are generated in columns that span the spinal cord and reach into the hindbrain, indicating that the mechanisms of dorsoventral patterning of the developing spinal cord and hindbrain are similar. Lbxl is expressed in the spinal cord and hindbrain, and our analyses show some similarities in Lbx1 function in these two units of the developing nervous system. In particular, two major neuronal classes (A and B) can be defined in the alar plate of both units. In the spinal cord and in the hindbrain of $L b x 1$ mutant mice, class B neurons assume the molecular characteristics of class A neurons. Thus, principal mechanisms of neuronal specification along the dorsoventral axis, as well as the principal Lbxl function in this process, appear to be conserved in the spinal cord and hindbrain.

dBLb neurons of rhombomere 7 resemble dILb neurons in the spinal cord: (1) dBLb and dILb neurons are born during the late neurogenic phase; (2) dBLb and dILb neurons express Lbx1, Lmx1b, and Tlx3; and (3) dBLb and dILb derivatives exhibit functional similarity, in that they process somatosensory information of the face and body, respectively; however, molecular and functional characteristics of the mis-specified $\mathrm{dBLb}^{*}$ and $\mathrm{dILb}^{\star}$ neurons of $\mathrm{Lb} \times 1$ mutants are distinct. $\mathrm{dBLb}^{*}$ neurons of rhombomere 7 express Phox $2 b, \operatorname{Lmx} 1 b$, and Tlx 3 and assume the molecular characteristics of viscerosensory relay neurons, whereas dILb* neurons of the spinal cord express Isl1/2 and Tlx3 and are thought to assume the molecular characteristics of neurons that process proprioceptive information (cf. Gross et al., 2002; Müller et al., 2002). Thus, despite the principal similarity of Lbxl functions in the spinal cord and hindbrain, additional regulatory mechanisms exist that distinguish the two units. Extensive studies showed differences in Hox gene expression along the anterior-posterior axis of the nervous system and the combinatorial function of Hox genes in the specification of anterior-posterior identity. Thus, mutations or misexpression of Hox genes can cause homeotic transformations of rhombomeres (Wilkinson et al., 1989; Hunt et al., 1991; Nonchev et al., 1997; Bell et al., 1999; Barrow et al., 2000; Trainor and Krumlauf, 2000; Gaufo et al., 2003; Kiecker and Lumsden, 2005). In addition, Hox genes can also affect the specification of discrete neuron types. For in- 
stance, in HoxB1 and HoxA3/HoxB3 mutant mice, a population of Phox $2 \mathrm{~b} / \mathrm{Lmx} 1 \mathrm{~b}$ dorsal neurons likely to correspond to viscerosensory relay neurons assume an aberrant fate and express Lbx1 in rhombomeres 4 and 5, respectively (Gaufo et al., 2004). The rhombomeres retained some normal characteristics in the mutants, indicating that these fate changes cannot be attributed solely to changes in rhombomere identity. Instead, these findings point toward a direct role of Hox genes in the specification of viscerosensory relay neurons by controlling the expression of Lbx1 and Phox2b.

\section{References}

Altman J, Bayer SA (1980) Development of the brain stem in the rat: I. Thymidine-radiographic study of the time of origin of neurons of the lower medulla. J Comp Neurol 194:1-35.

Barrow JR, Stadler HS, Capecchi MR (2000) Roles of Hoxa1 and Hoxa2 in patterning the early hindbrain of the mouse. Development 127:933-944.

Bell E, Wingate RJ, Lumsden A (1999) Homeotic transformation of rhombomere identity after localized Hoxb1 misexpression. Science 284:2168-2171.

Bergquist H, Kallen B (1955) The archencephalic neuromery in Ambystoma punctatum: an experimental study. Acta Anat (Basel) 24:208-214.

Blessing WW (1997) The lower brainstem and bodily homeostasis. New York: Oxford UP.

Briscoe J, Ericson J (2001) Specification of neuronal fates in the ventral neural tube. Curr Opin Neurobiol 11:43-49.

Brunet JF, Pattyn A (2002) Phox2 genes: from patterning to connectivity. Curr Opin Genet Dev 12:435-440.

Cambronero F, Puelles L (2000) Rostrocaudal nuclear relationships in the avian medulla oblongata: a fate map with quail chick chimeras. J Comp Neurol 427:522-545.

Caspary T, Anderson KV (2003) Patterning cell types in the dorsal spinal cord: what the mouse mutants say. Nat Rev Neurosci 4:289-297.

Cheng L, Arata A, Mizuguchi R, Qian Y, Karunaratne A, Gray PA, Arata S, Shirasawa S, Bouchard M, Luo P, Chen CL, Busslinger M, Goulding M, Onimaru H, Ma Q (2004) Tlx3 and Tlxl are post-mitotic selector genes determining glutamatergic over GABAergic cell fates. Nat Neurosci 7:510-517.

Dauger S, Pattyn A, Lofaso F, Gaultier C, Goridis C, Gallego J, Brunet JF (2003) Phox $2 \mathrm{~b}$ controls the development of peripheral chemoreceptors and afferent visceral pathways. Development 130:6635-6642.

Farago AF, Awatramani RB, Dymecki SM (2006) Assembly of the brainstem cochlear nuclear complex is revealed by intersectional and subtractive genetic fate maps. Neuron 50:205-218.

Farley FW, Soriano P, Steffen LS, Dymecki SM (2000) Widespread recombinase expression using FLPeR (flipper) mice. Genesis 28:106-110.

Gaufo GO, Thomas KR, Capecchi MR (2003) Hox3 genes coordinate mechanisms of genetic suppression and activation in the generation of branchial and somatic motoneurons. Development 130:5191-5201.

Gaufo GO, Wu S, Capecchi MR (2004) Contribution of Hox genes to the diversity of the hindbrain sensory system. Development 131:1259-1266.

Gross MK, Dottori M, Goulding M (2002) Lbxl specifies somatosensory association interneurons in the dorsal spinal cord. Neuron 34:535-549.

Hippenmeyer S, Vrieseling E, Sigrist M, Portmann T, Laengle C, Ladle DR, Arber S (2005) A developmental switch in the response of DRG neurons to ETS transcription factor signaling. PLoS Biol 3:e159.

Hunt P, Gulisano M, Cook M, Sham MH, Faiella A, Wilkinson D, Boncinelli E, Krumlauf R (1991) A distinct Hox code for the branchial region of the vertebrate head. Nature 353:861-864.

Jagla K, Dolle P, Mattei MG, Jagla T, Schuhbaur B, Dretzen G, Bellard F, Bellard M (1995) Mouse Lbxl and human LBX1 define a novel mam- malian homeobox gene family related to the Drosophila lady bird genes. Mech Dev 53:345-356.

Jessell TM (2000) Neuronal specification in the spinal cord: inductive signals and transcriptional codes. Nat Rev Genet 1:20-29.

Kiecker C, Lumsden A (2005) Compartments and their boundaries in vertebrate brain development. Nat Rev Neurosci 6:553-564.

Landsberg RL, Awatramani RB, Hunter NL, Farago AF, DiPietrantonio HJ, Rodriguez CI, Dymecki SM (2005) Hindbrain rhombic lip is comprised of discrete progenitor cell populations allocated by Pax6. Neuron 48:933-947.

Lee KJ, Jessell TM (1999) The specification of dorsal cell fates in the vertebrate central nervous system. Annu Rev Neurosci 22:261-294.

Machold R, Fishell G (2005) Math1 is expressed in temporally discrete pools of cerebellar rhombic-lip neural progenitors. Neuron 48:17-24.

Marin F, Puelles L (1995) Morphological fate of rhombomeres in quail/ chick chimeras: a segmental analysis of hindbrain nuclei. Eur J Neurosci 7:1714-1738.

Müller T, Brohmann H, Pierani A, Heppenstall PA, Lewin GR, Jessell TM, Birchmeier C (2002) The homeodomain factor Lbxl distinguishes two major programs of neuronal differentiation in the dorsal spinal cord. Neuron 34:551-562.

Nonchev S, Maconochie M, Gould A, Morrison A, Krumlauf R (1997) Cross-regulatory interactions between Hox genes and the control of segmental expression in the vertebrate central nervous system. Cold Spring Harb Symp Quant Biol 62:313-323.

Pattyn A, Morin X, Cremer H, Goridis C, Brunet JF (1999) The homeobox gene Phox $2 \mathrm{~b}$ is essential for the development of autonomic neural crest derivatives. Nature 399:366-370.

Pattyn A, Goridis C, Brunet JF (2000) Specification of the central noradrenergic phenotype by the homeobox gene Phox $2 \mathrm{~b}$. Mol Cell Neurosci 15:235-243.

Pattyn A, Guillemot F, Brunet JF (2006) Delays in neuronal differentiation in Mash1/Ascl1 mutants. Dev Biol 295:67-75.

Qian Y, Fritzsch B, Shirasawa S, Chen CL, Choi Y, Ma Q (2001) Formation of brainstem (nor)adrenergic centers and first-order relay visceral sensory neurons is dependent on homeodomain protein Rnx/Tlx3. Genes Dev 15:2533-2545.

Qian Y, Shirasawa S, Chen CL, Cheng L, Ma Q (2002) Proper development of relay somatic sensory neurons and D2/D4 interneurons requires homeobox genes Rnx/Tlx-3 and Tlx-1. Genes Dev 16:1220-1233.

Rodriguez CI, Buchholz F, Galloway J, Sequerra R, Kasper J, Ayala R, Stewart AF, Dymecki SM (2000) High-efficiency deleter mice show that FLPe is an alternative to Cre-loxP. Nat Genet 25:139-140.

Schubert FR, Dietrich S, Mootoosamy RC, Chapman SC, Lumsden A (2001) Lbx1 marks a subset of interneurons in chick hindbrain and spinal cord. Mech Dev 101:181-185.

Trainor PA, Krumlauf R (2000) Patterning the cranial neural crest: hindbrain segmentation and Hox gene plasticity. Nat Rev Neurosci 1:116-124.

Vaage S (1969) The segmentation of the primitive neural tube in chick embryos (Gallus domesticus): a morphological, histochemical and autoradiographical investigation. Ergeb Anat Entwicklungsgesch 41:3-87.

Vasyutina E, Stebler J, Brand-Saberi B, Schulz S, Raz E, Birchmeier C (2005) CXCR4 and Gab1 cooperate to control the development of migrating muscle progenitor cells. Genes Dev 19:2187-2198.

Wang VY, Rose MF, Zoghbi HY (2005) Math1 expression redefines the rhombic lip derivatives and reveals novel lineages within the brainstem and cerebellum. Neuron 48:31-43.

Wilkinson DG, Bhatt S, Cook M, Boncinelli E, Krumlauf R (1989) Segmental expression of Hox-2 homeobox-containing genes in the developing mouse hindbrain. Nature 341:405-409. 